\title{
Cluster Analysis of Red Rice based on SSR Markers from Hani's Terraced Fields in Yunnan Province
}

\author{
Mengli Ma, Yanhong Liu, Tiantao Wang and Bingyue Lu ${ }^{a}$ \\ College of Life Science and Technology/ Key Laboratory of Crop High Quality and Efficient \\ Cultivation and Security Control of College in Yunnan Province, Honghe University, Yunnan Mengzi \\ 661100, China \\ a Corresponding author: Iby202@126.com
}

Keywords: Red rice; Hani's terraces fields; Genetic diversity.

\begin{abstract}
Genetic diversity is the main source of variability in any crop improvement program. There are abundant rice landraces in Hani's terraces fields in Yunnan, especially red rice resources. A set of 61 red rice landraces were characterized using 78 simple sequence repeat (SSR) markers. Cluster analysis based on unweighted pair group method with arithmetic mean showed that the similarity coefficients varied from 0.19 to 0.85 , all genotypes grouped into two major clusters in the dendrogram at 0.19 similarity. Indica rice clusters including 58 rice landraces as main rice cultivation types in Hani's terraces fields in Yunnan.
\end{abstract}

\section{Introduction}

Rice (Oryza sativa L.) is the most essential and important food crop of the world, particularly in Asia. Genetic diversity is an important basis for rice genetic improvement. Yunnan province is one of the world's recognized centers of genetic diversity and origin of cultivated rice in Asia, is the largest genetic and ecological diversity center of rice germplasm resources in China [1]. The Yuanyang Hani's terraces are located at $22^{\circ} 49^{\prime}-23^{\circ} 19^{\prime} \mathrm{N}, 102^{\circ} 27^{\prime}-103^{\circ} 13^{\prime} \mathrm{E}$. The environment of the uplands is suitable for rice cultivation with average annual sunshine of 1670 hours and an average temperature of $15.4^{\circ} \mathrm{C}$. The Hani's people live on a hillside at an altitude between $1400-2000 \mathrm{~m}$. They have farmed rice in the terraced fields for over 1500 years [2]. A rich diversity of rice varieties thrives there and the local people retain the tradition of seed exchange and rice worship [3]. Grain quality currently represents a major problem in rice production in China and many other rice producing areas of the world [4]. Since a long time ago, Hani's people have believed that the traditional red rice varieties are superior in nutritional quality and healthy effect. The recent studies showed the red rice is superior in antioxidant capacity and nutritional quality $[5,6]$. Simple Sequence repeat (SSR) markers have been commonly used in genetic diversity studies in rice because of high level of polymorphism which helps to establish the relationship among the individuals even with less number of markers [7]. The aim of this study is to understand the classification of rice landraces in Hani's terraces and provide theoretical basis for protection and utilization of rice resources.

\section{Materials and Methods}

\subsection{Rice materials}

A collection consisting of 61 red rice landraces was used in this study, which collected from twelve different township of Yuanyang County, Yunnan Province, China as well as from Daping Township, Eza Township, Ganiang Township, Huangcaoling Township, Huangmaoling Township, Niujiaozhai Township, Panzhihua Township, Shalatuo Township, Shangxincheng Township, Shengcun Township, Xiaoxinjie Township, Xinjie Town, 12 varieties origin is unknown (Table 1). 
Table 1 Name and collecting sites of red rice landraces in this study.

\begin{tabular}{|c|c|c|}
\hline Name of red rice landraces & $\begin{array}{c}\text { Number of } \\
\text { Varieties }\end{array}$ & Collection site \\
\hline cesuogu, honggu2, honggu3 & 3 & Daping Township \\
\hline dalonggu, maichou, helingu, wupucheni & 4 & Eza Township \\
\hline honggu1 & 1 & Ganiang Township \\
\hline laojinghongmi, shiouqian, chenilongge, apenpenche & 4 & Huangcaoling Township \\
\hline anxinggu, huagu1, shuihangu & 3 & Huangmaoling Township \\
\hline rongrenche, rongrenrenche, luoge, chemanche,maoche & 5 & Niujiaozhai Township \\
\hline laopinzhonghongmi, laopinzhong & 2 & Panzhihua Township \\
\hline dianmeng2hao, honggunuo & 2 & Shalatuo Township \\
\hline $\begin{array}{c}\text { dumiaogu, honggunuo, gaoshanhonggu1, taiyanggu, } \\
\text { bendihonggu2, laomujigu }\end{array}$ & 6 & Shangxincheng Township \\
\hline chenu, huagu2 & 2 & Shengcun Township \\
\hline $\begin{array}{c}\text { bianhao3, poxinggu } \\
\text { baijiaolaojing,yingu, lvjiaogu, longshanlinggu, } \\
\text { aizheranche, chefu, liuyuegu,cheran, chejiagu, } \\
\text { yuelianggu }\end{array}$ & 2 & Xiaoxinjie Township \\
\hline $\begin{array}{c}\text { chenal,chemaouzheng, chejiaojiaoer, bendihonggu1, } \\
\text { gutianche,gaoshanhonggu2, pozhugu, chengke, jiagu, } \\
\text { chengni, cheniu, zajiaohonggu }\end{array}$ & 12 & Xinjie Town \\
\hline
\end{tabular}

\subsection{DNA template preparation and PCR}

DNA was extracted using a protocol adapted from Dellaporta et al. [8]. Following solubilization of the DNA in TE buffer, its concentration was assessed spectrophotometrically, and this was used to prepare working solutions of $20 \mathrm{ng} / \mathrm{ul}$. The PCR analysis was carried out based on SSR primers documented in the Gramene database (www.gramene.org), and the amplification protocols followed Chen et al. [9] with only minor modifications. Briefly, each $10 \mu 1$ reaction contained 10ng template DNA, $0.2 \mu \mathrm{M}$ of each primer, $2.5 \mathrm{mM}$ dNTP, $1 \mu 110 \mathrm{x} \mathrm{Mg}^{2+}$ free buffer, $25 \mathrm{mM} \mathrm{MgCl}_{2}$ and $0.5 \mathrm{U}$ rTaq DNA polymerase (Sangon Biotech, CHN). The cycling regime consisted of a denaturation step $\left(95^{\circ} \mathrm{C} / 5 \mathrm{~min}\right)$, followed by 30 cycles of $95^{\circ} \mathrm{C} / 30 \mathrm{~s}, 55-58^{\circ} \mathrm{C} / 30 \mathrm{~s}, 72^{\circ} \mathrm{C} / 30 \mathrm{~s}$, and a final extension step of $72^{\circ} \mathrm{C} / 10 \mathrm{~min}$. The amplicons were electrophoretically separated through $8 \%$ non-denaturing polyacrylamide gels, and visualized by silver staining [10].

\subsection{Data analysis}

Amplified fragments of different sizes were considered as different alleles. DNA bands that were amplified by a given primer were scored as present (1) or absent (0) for all the samples under study. Genetic similarities based on the Dice coefficient were calculated among all possible pairs with the SIMQUAL option and organized in a similarity matrix using NTSYS-pc version 2.10e package[11] and this similarity matrix was used in cluster analysis using an unweighted pair-group method with arithmetic averages (UPGMA) and sequential, agglomerative, hierarchical and nested (SAHN) clustering algorithm to obtain a dendrogram.

\section{Results}

The genetic relationships among rice genotypes are presented in a dendrogram using UPGMA-based clustering analysis with the Dice similarity coefficient (Fig. 1). The genetic similarity coefficient of 61 rice landraces was $0.19 \sim 0.85$. All genotypes clearly grouped into two major clusters in the dendrogram at 0.19 similarity. The first cluster (I) represents the Indica rice including 58 rice landraces, while the second cluster (II) represents the japonica including 3 rice landraces. The indica cluster was divided into two subgroups with a similarity coefficient of 0.42 . 
Sub-cluster I-A comprised of 57 rice landraces, whereas sub-cluster I-B comprised of one rice landraces, Honggu3. As the main sub-cluster, when the genetic similarity coefficient of 0.51 as the threshold, I-A was divided into I-A1 (54 rice landraces) and I-A2 (3 rice landraces), when the genetic similarity coefficient of 0.53 as the threshold, I-A1 was further divided into two subgroups, I-A1a (12 rice landraces) and I-A1b (42 rice landraces). The genetic similarity coefficient between lvjiaogu and lalonggu was the highest, 0.85 , in addition to liuyuegu and lhuihangu, laomujigu and lajiaohonggu also had high genetic similarity.

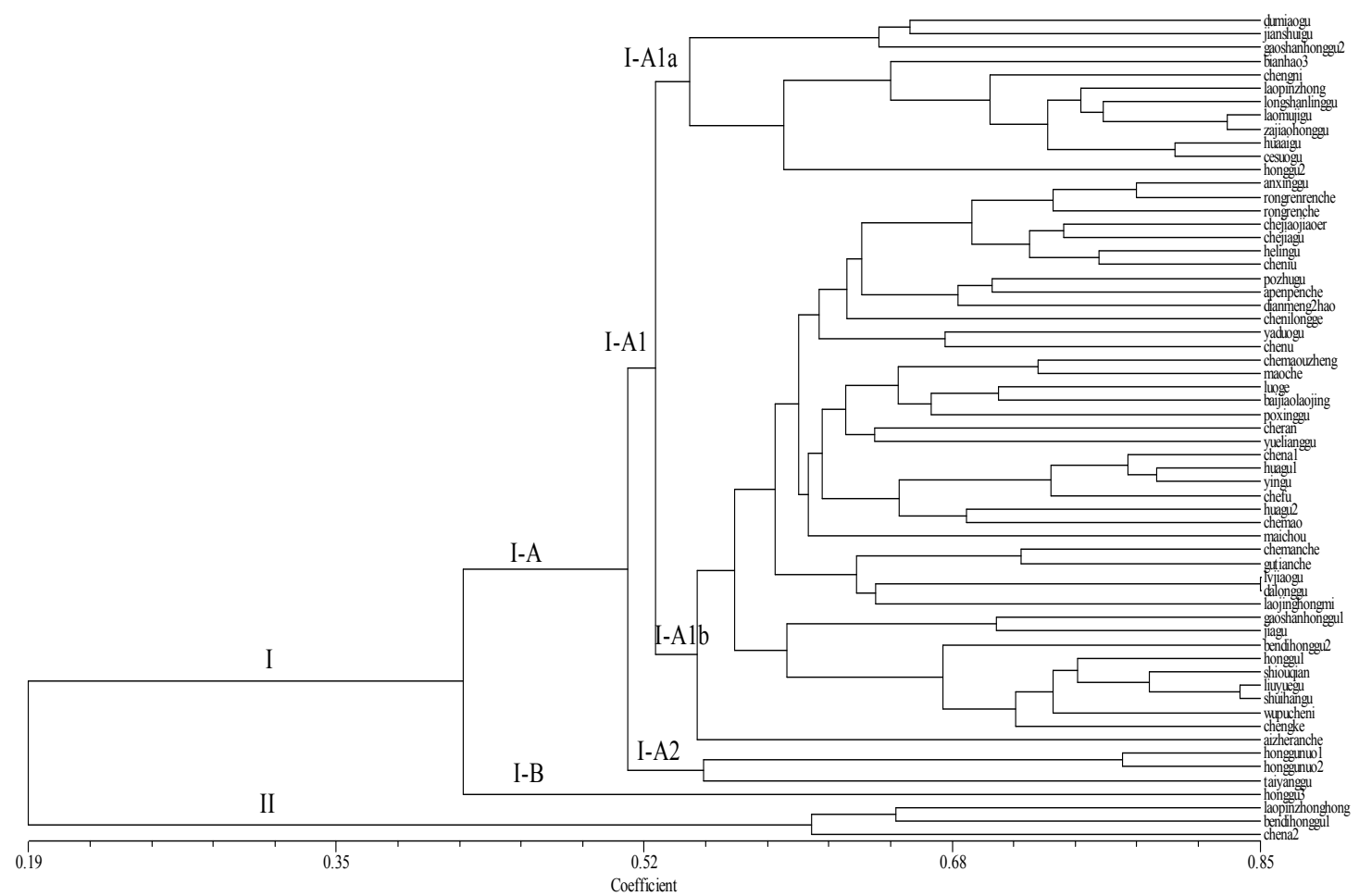

Fig. 1 Unweighted pair group method with arithmetic mean (UPGMA) based dendrogram of rice genotypes using simple sequence repeat (SSR) markers.

\section{Discussion}

In the present study, 61 red rice landraces were grouped into two clusters based on their similarity coefficients. 58 rice landraces were divided into Indica cluster (I) and 3 rice landraces were divided into japonica cluster (II). The indica was a majority variety types in Hani 's Terraced, is similar to earlier studies [12-15], there were still a great deal of genetic differentiation in indica cluster, including different subgroups(Fig. 1). The phenomenon "different varieties of the same name" and "same varieties of different name" also exists in our study, for example honggu, chena, huagu, bendihonggu and gaoshanhonggu were clustered under different groups respectively, while lvjiaogu and dalonggu was clustered together at a similarity coefficient of 0.85 . This is mainly because seed exchange and variety rotation were widely used by the Hani people in Yuanyang County. In conclusion, SSR marker based molecular characterization of Hani's red rice landraces revealed that large variation exists among the accessions. This study will also provide a theoretical basis for the protection and utilization of these rice resources.

\section{Acknowledgments}

This paper is funded by Fundamental Research Program of Yunnan Province (2013FZ124), Yunnan Provincial Department of Education Science Research Fund Project (2013Y066) and Honghe University youth backbone of academic training project (2014GG0101). 


\section{References}

[1] Zeng Y, Zhang H, Li Z, et al. Evaluation of genetic diversity of rice landraces (Oryza sativa L.) in Yunnan, China[J]. Breeding Science, 2007, 57(2): 91-99.

[2] Wang Q. On the culture of the terraced fields. Yunnan University Press, 1999, 47-50. (In Chinese).

[3] Xu F R, Tang C F, Yu T Q, et al. Diversity of paddy rice varieties from Yuanyang Hani's terraced fields in Yunnan, China[J]. Acta Ecologica Sinica, 2010, 30(12): 3346-3357.

[4] Tan Y F, Li J X, Yu S B, et al. The three important traits for cooking and eating quality of rice grains are controlled by a single locus in an elite rice hybrid, Shanyou 63[J]. Theoretical and Applied Genetics, 1999, 99(3-4): 642-648.

[5] Gunaratne $\mathrm{A}, \mathrm{Wu} \mathrm{K}, \mathrm{Li} \mathrm{D}$, et al. Antioxidant activity and nutritional quality of traditional red-grained rice varieties containing proanthocyanidins[J]. Food chemistry, 2013,138 (2): 1153 $-1161$.

[6] Sumczynski D, Kotásková E, Družbíková H, et al. Determination of contents and antioxidant activity of free and bound phenolics compounds and in vitro digestibility of commercial black and red rice (Oryza sativa L.) varieties[J]. Food Chemistry, 2016, 211: 339-346.

[7] McCouch S R, Chen X, Panaud O, et al. Microsatellite marker development, mapping and applications in rice genetics and breeding[J]. Plant molecular biology, 1997, 35(1-2): 89-99.

[8] Dellaporta SL, Wood J, Hicks JB (1983) A plant DNA minipreparation: Version II. Plant Mol Biol Rep 1:19-21.

[9] Chen X, Temnykh S, Xu Y, et al. Development of a microsatellite framework map providing genomewide coverage in rice (Oryza sativa L.). Theor Appl Genet, 1997, 95:553-567.

[10] Sanguinetti CJ, Dias NE, Simpson AJG. Rapid silver staining and recover of PCR products separated on polyacrylamide gels. Biotechnology,1994, 17:915-919.

[11]Rohlf, F. NTSYS-pc Numerical Taxonomy and Multivariate Analysis System; Exeter Software: New York, NY, USA, 2001.

[12]Liu C C, Zhao F W, Wu X X, et al. Genetic diversity and population structure analysis of currently cultivated rice landraces from Hani's Terraced Fields in Yunnan Province moleal. Chin J Rice Sci, 2015, 29(1):28-34. (in Chinese with English abstract)

[13]Zhang H, Sun J, Wang M, et al. Genetic structure and phylogeography of rice landraces in Yunnan, China, revealed by SSR[J]. Genome, 2006, 50(1): 72-83.

[14]Zhu M Y, Wang Y Y, Zhu Y Y, et al. Estimating genetic diversity of rice landraces from Yunnan by SSR assay and its implication for conservation[J]. Acta Bot Sin, 2004, 46(12): 1458-1467.

[15] Gao D, Li R, Yang M Q, et al. Rice landraces' genetic diversity of Hani terrace wetland in Qingkou village of Yuanyang county. Molecular Plant Breeding, 2011, 9 (118): 1857-1863. (in Chinese with English abstract) 